\title{
Spectral and Photophysical Characterization of Donor- $\pi$-Acceptor Arylthienyl- and Bithienyl-Benzothiazole Derivatives in Solution and Solid State
}

\author{
João Pina, ${ }^{\dagger}$ J. Sérgio Seixas de Melo, ${ }^{*, \dagger}$ Hugh D. Burrows, ${ }^{\dagger}$ Rosa M. F. Batista,,$^{\dagger}$ \\ Susana P. G. Costa, $\$$ and M. Manuela M. Raposo \\ Departament of Chemistry, University of Coimbra, Rua Larga, 3004-535 Coimbra, Portugal, and Centro de \\ Química, Universidade do Minho, Campus de Gualtar, 4710-057, Braga, Portugal
}

Received: April 20, 2007; In Final Form: June 20, 2007

\begin{abstract}
A comprehensive study has been made in solution at room temperature ( $293 \mathrm{~K})$, low temperature $(77 \mathrm{~K})$, and in thin films (Zeonex matrixes) of the spectral and photophysical properties of six arylthienyl- and bithienylbenzothiazole derivatives functionalized with different donor groups. Similar experiments have been carried out with two related precursors (containing the arylthienyl and aryl-bithienyl conjugated systems), and results are compared. Singlet-singlet and triplet-triplet absorption spectra, emission spectra together with lifetimes and quantum yields have been obtained, and from these data the rates for all the radiative and nonradiative processes determined, providing information on the dominant decay processes. The arylthienyl-benzothiazole derivatives show high fluorescence quantum yields $\left(\phi_{\mathrm{F}}\right)$ with negligible internal conversion $\left(\phi_{\mathrm{IC}}\right)$, whereas the bithienyl-benzothiazoles display lower but still significant $\phi_{\mathrm{F}}$ values, but now radiationless processes $\left(\phi_{\mathrm{IC}}\right.$ and $\left.\phi_{\text {ISC }}\right)$ are competitive. A comparison with the analogous oligothiophenes is made. Singlet oxygen yields were also determined and the triplet energy transfer to ${ }^{3} \mathrm{O}_{2}$ to produce ${ }^{1} \mathrm{O}_{2}$ was found to be highly efficient with values of $\mathrm{S}_{\Delta}\left(=\phi_{\Delta} / \phi_{\mathrm{T}}\right)$ varying from 0.4 to 1 .
\end{abstract}

\section{Introduction}

Conjugated polymers and oligomers are among the most important advanced materials currently under study, and a significant number of real and potential applications have been demonstrated. Recent predictions suggest that the present global market for these materials will increase from the current level of $\$ 1-2$ billion/year to over $\$ 100$ billion/year by $2020 .{ }^{1}$ Among those applications that have proved most promising are their uses in photovoltaic cells, ${ }^{2,3}$ field effect transistors (FETs), and in light-emitting diodes (LEDs). ${ }^{4,5}$ In the latter cases, it is common to differentiate between low-molecular-weight (oligomeric and small molecule) and high-molecular-weight (polymeric) systems. In part, this stems from the differences in methods of device preparation with the two classes of materials. However, there is frequently a relationship between the properties of these two groups, that is, between the polymers and their respective oligomers. ${ }^{6}$ Among the LED polymers (and oligomers) which have received particular attention, fluorene-, p-phenylenevinylene (PPV)-, and thiophene-based systems have proved to be particularly interesting. ${ }^{7-11}$ One of the major directions of current research on OLEDs involves the improvement of the emissive properties. This can be done by enhancing the fluorescence properties (for example, by adding strongly emissive groups by producing a copolymer) or by capturing the normally nonradiative triplet state with lower-energy phosphorescent molecules, for example, metalloporphyrins or iridium(III) complexes, which can then luminesce. ${ }^{12}$ The benzothiazole group is known to be a highly fluorescent chromophore and its free base recently and metal complexes have been widely investigated recently as electron-transporting and

* Corresponding author. Fax: 00351239 827703. E-mail: sseixas@ci.uc.pt.

$\dagger$ University of Coimbra.

$\doteqdot$ Universidade do Minho. -emitting layers for OLEDs. ${ }^{13,14}$ The link with the well-known oligothiophenes constitutes a useful approach in order to improve the luminescent properties of a conjugated organic oligomer (and polymer). In general, the oligothiophenes have the advantage of being more easily soluble in organic solvents, facilitating thin-film preparation by solvent-based techniques, in addition to facilitating tuning the emission spectra by chemical modifications of their structure.

Recently, the synthesis, the solvatochromic behavior, the nonlinear optical properties, and some fluorescence properties of several substituted arylthienyl, bithienyl derivatives and conjunction of these with the benzothiazole unit were described. ${ }^{15-19}$ In this work, we extend these preliminary fluorescence studies ${ }^{16,18}$ to a comprehensive and complete spectral (singlet and triplet state absorption maxima) and photophysical study of these compounds in solution (at room and low temperatures) and in solid matrixes (thin films).

In the present study, electron-donor (D) groups are linked through a $\pi$-conjugated link to an electron-acceptor (A) group, giving the overall chromophore D- $\pi$-A character. From Scheme 1 , the structures of the $\mathrm{D}$ groups investigated are $\mathrm{NMe}_{2}, \mathrm{OMe}$, and $\mathrm{OEt}$; the $\pi$-conjugated bridge involves the arylthienyl or bithienyl systems; and the acceptor is the $\pi$-electron-deficient benzothiazole heterocycle. The presence of electron-donor and -acceptor groups in these compounds also makes these derivatives interesting for nonlinear optical (NLO) applications. ${ }^{15,17,19-22}$ Although there exists strong interest in the NLO applications for this type of derivatives (oligothienyl-substituted benzazoles), the present work focuses on the comprehensive study of the photophysical properties.

Substitution of the bithiophene chromophore by the more electron-rich moiety $3^{\prime}, 4^{\prime}$-(ethylenedioxy)-2,2'-bithiophene (see Scheme 1, BZT2d) was also investigated. The changes induced by the incorporation of electron-acceptor groups are likely to 


\section{SCHEME 1}

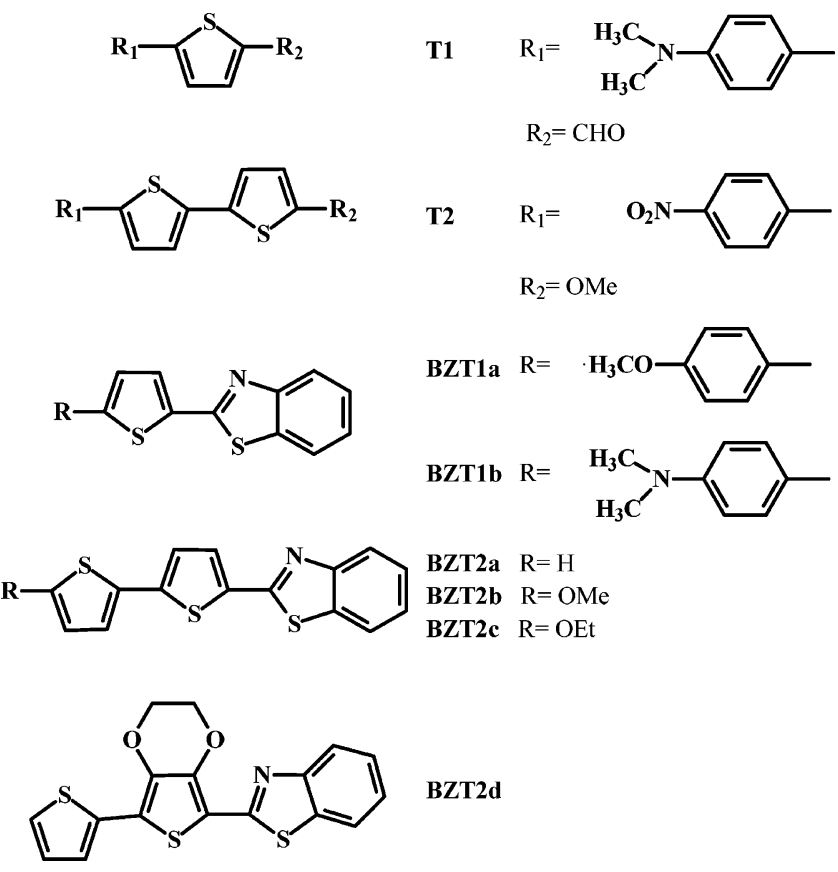

lower the energy of the LUMO orbital, while the electron-donor side-chain groups will act over the HOMO orbital and, as a consequence, change the HOMO - LUMO $(\Delta E)$ separation. ${ }^{22,23}$

\section{Experimental Section}

Absorption and fluorescence spectra were recorded on Shimadzu UV-2100 and Horiba-Jobin-Ivon SPEX Fluorog 3-22 spectrometers, respectively. The fluorescence spectra were corrected for the wavelength response of the system.

The fluorescence quantum yields were measured using quaterthiophene $\left(\phi_{\mathrm{F}}=0.18\right.$ in ethanol $),{ }^{24}$ quinquethiophene $\left(\phi_{\mathrm{F}}\right.$ $=0.33$ in $\mathrm{MCH}),{ }^{24}$ and quinine sulfate $\left(\phi_{\mathrm{F}}=0.54\right.$ in $0.5 \mathrm{M}$ $\left.\mathrm{H}_{2} \mathrm{SO}_{4}\right)^{25}$ as standards.

Fluorescence decays were measured using a home-built TCSPC apparatus elsewhere described ${ }^{26}$ and were analyzed using the modulating functions method of Striker. ${ }^{27}$

The experimental setup used to obtain triplet spectra and triplet yields has been described elsewhere. ${ }^{26,28}$ First-order kinetics were observed for the decay of the lowest triplet state. Solutions of the compounds in ethanol were pumped through a $10 \mathrm{~mm}$ thick cell at $1 \mathrm{~mL} / \mathrm{min}$ flow rate using an SSI chromatography pump. They were then irradiated with a 355 $\mathrm{nm}$ unfocused beam. Special care was taken in determining triplet yields to have optically matched dilute solutions (abs $\approx$ 0.2 in a $10 \mathrm{~mm}$ square cell) and low laser energy $(\leq 2 \mathrm{~mJ})$ to avoid multiphoton and $\mathrm{T}-\mathrm{T}$ annihilation effects.

The triplet molar absorption coefficients were obtained by the singlet depletion and energy transfer methods. Details on the experimental procedures and equations used can be found in ref 26,28 and in the Supporting Information section.

Room-temperature singlet oxygen phosphorescence was detected at $1270 \mathrm{~nm}$ with an equipment and procedures elsewhere reported. ${ }^{26,28}$ From these signals the singlet oxygen quantum yields were obtained. The procedures can be found in detail in the Supporting Information section.

Thin films from the compounds were obtained with a DeskTop Precision Spin Coating System, Model P6700 Series from Speedline Technologies with procedures reported elsewhere. ${ }^{26,28}$ The fluorescence emission spectra of the thin films were

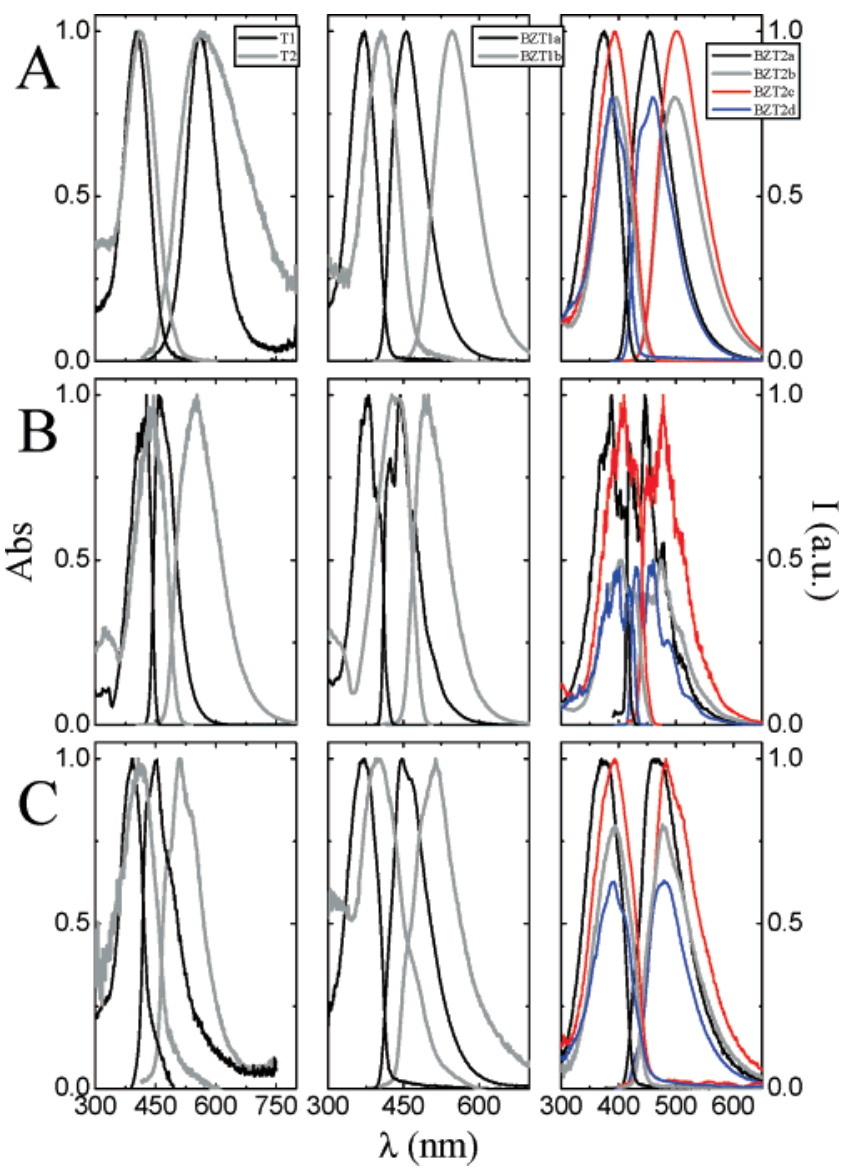

Figure 1. Absorption and fluorescence spectra for the arylthienyl- and bithienyl-benzothiazoles in ethanol solution at (A) room (293 K), (B) low temperature (from excitation spectra in the case of absorption), and $(\mathrm{C})$ in films.

obtained with a Horiba-Jobin-Yvon integrating sphere. The solid-state photoluminescence quantum yields in thin films were obtained as described elsewhere (see Supporting Information section for further details)

\section{Results and Discussion}

The structures and acronyms of the compounds studied are shown in Scheme 1. We are interested in two main issues in the present study: (1) the influence of the addition of a benzothiazole group on the photophysical properties of arylthienyl and bithienyl moieties, and (2) the effect on these of donor groups in the terminal positions of the thienyl or on the aryl moieties.

In order to get a complete picture of the photophysical behavior of these compounds, a comprehensive study has been performed involving determination of the singlet-singlet absorption, fluorescence emission and triplet-triplet absorption spectra, together with quantum yields of fluorescence, intersystem crossing, internal conversion and singlet oxygen formation, and singlet and triplet state lifetimes.

Absorption and Fluorescence Spectra.

Figure 1 depicts the absorption spectra and fluorescence of the compounds in Scheme 1 obtained in ethanol solution at room and low temperatures, and in thin films. From the room temperature solution absorption spectra (see Figure 1 and Table 1), a red-shift is seen as we go through the series $\mathrm{BZT} 1 \mathrm{a} \approx \mathrm{BZT} 2 \mathrm{a}<\mathrm{BZT} 2 \mathrm{~b} \approx \mathrm{BZT} 2 \mathrm{c} \approx \mathrm{BZT} 2 \mathrm{~d}<\mathrm{T} 1 \approx$ BZT1b $<$ T2. 
TABLE 1: Spectral Data (absorption, emission wavelength maxima and triplet maxima and extinction coefficients) for the Arylthienyl- and Bithienyl-Benzothiazoles in Ethanol at Room Temperature (293 K), Low Temperature (77 K), and in Films

\begin{tabular}{|c|c|c|c|c|c|c|c|c|c|c|c|}
\hline compound & $\begin{array}{c}\lambda_{\max }^{\mathrm{abs}} \\
(\mathrm{nm}) \\
293 \mathrm{~K} \\
\end{array}$ & $\begin{array}{l}\lambda_{\max }^{\mathrm{abs}} \\
(\mathrm{nm}) \\
77 \mathrm{~K} \\
\end{array}$ & $\begin{array}{l}\lambda_{\max }^{\mathrm{abs}} \\
(\mathrm{nm}) \\
\text { film }\end{array}$ & $\begin{array}{r}\lambda_{\max }^{\text {fluo }} \\
(\mathrm{nm}) \\
293 \mathrm{~K} \\
\end{array}$ & $\begin{array}{l}\lambda_{\max }^{\text {fluo } b} \\
(\mathrm{~nm}) \\
77 \mathrm{~K} \\
\end{array}$ & $\begin{array}{l}\lambda_{\max }^{\text {fluo }} \\
(\mathrm{nm}) \\
\text { film }\end{array}$ & $\lambda_{\max }^{\mathrm{T}_{1} \rightarrow \mathrm{T}_{\mathrm{n}}}$ & $\begin{array}{c}\epsilon_{\mathrm{SS}} \\
\left(\mathrm{M}^{-1} \mathrm{~cm}^{-1}\right)\end{array}$ & $\begin{array}{c}\epsilon_{\mathrm{TT}} \\
\left(\mathrm{M}^{-1} \mathrm{~cm}^{-1}\right)\end{array}$ & $\begin{array}{c}\Delta_{\mathrm{SS}} \\
\left(\mathrm{cm}^{-1}\right) \\
293 \mathrm{~K}\end{array}$ & $\begin{array}{c}\Delta \\
(\mathrm{nm}) \\
\mathrm{S}_{1}-\mathrm{T}_{1} \rightarrow \mathrm{T}_{\mathrm{n}}\end{array}$ \\
\hline T1 & 404 & 425 & 390 & 562 & 460 & 450 & 620 & 25880 & 20860 & 6957 & 216 \\
\hline $\mathbf{T} 2$ & 413 & 442 & 413 & 562 & 550 & 510 & 665 & 14900 & 10585 & 6419 & 252 \\
\hline BZT1a & 370 & 378 & 370 & 455 & 443 & 446 & 530 & 17220 & 25290 & 5049 & 160 \\
\hline BZT1b & 407 & 435 & 400 & 547 & 495 & 514 & 520 & 18450 & $37570^{a}$ & 6288 & 113 \\
\hline BZT2a & 375 & 387 & 375 & 454 & $419,447,477$ & 467 & 500 & 35840 & 46850 & 4640 & 125 \\
\hline BZT2b & 395 & 405 & 394 & 498 & 450,473 & 478 & 475 & 26915 & $20650^{a}$ & 5236 & 80 \\
\hline BZT2c & 395 & 408 & 394 & 502 & 450,477 & 482 & 475 & 31620 & $22105^{a}$ & 5396 & 80 \\
\hline BZT2d & 388 & 400 & 391 & 460 & $430,458,485$ & 480 & 500 & 21750 & 33760 & 4034 & 112 \\
\hline
\end{tabular}

${ }^{a}$ In MCH. ${ }^{b}$ The italic values are the wavelength maxima.

TABLE 2: Photophysical Parameters (quantum yields and lifetimes) for the Arylthienyl- and Bithienyl-Benzothiazoles in Ethanol at Room Temperature $(293 \mathrm{~K})$, Low Temperature $(77 \mathrm{~K})$, and in Films

\begin{tabular}{|c|c|c|c|c|c|c|c|c|c|}
\hline compound & $\begin{array}{c}\phi_{\mathrm{F}} \\
(293 \mathrm{~K})\end{array}$ & $\begin{array}{c}\phi_{\mathrm{F}} \\
(77 \mathrm{~K})\end{array}$ & $\begin{array}{c}\phi_{\mathrm{F}} \\
(\text { film })\end{array}$ & $\begin{array}{c}\tau_{\mathrm{F}} \\
(\mathrm{ns})\end{array}$ & $\begin{array}{c}\tau_{\mathrm{F}} \\
(\mathrm{film})(\mathrm{ns})\end{array}$ & $\phi_{\mathrm{T}}$ & $\begin{array}{c}\tau_{\mathrm{T}} \\
(\mu \mathrm{s})\end{array}$ & $\phi_{\text {IC }}$ & $\phi_{\Delta}$ \\
\hline T1 & 0.070 & 0.66 & 0.11 & 1.03 & 3.76 & 0.15 & 1.52 & 0.78 & 0.061 \\
\hline $\mathbf{T} 2$ & 0.0038 & 0.33 & 0.27 & 2.04 & 3.02 & 0.11 & 1.04 & 0.89 & 0.11 \\
\hline BZT1a & 0.78 & 0.53 & 0.28 & 2.15 & 2.24 & 0.18 & 49.2 & 0.040 & 0.091 \\
\hline BZT1b & 0.84 & 0.51 & 0.12 & 2.91 & 2.45 & 0.094 & 1.94 & 0.066 & 0.090 \\
\hline BZT2a & 0.31 & 0.42 & 0.22 & 1.19 & 2.54 & 0.52 & 1.31 & 0.17 & 0.53 \\
\hline BZT2b & 0.65 & 0.44 & 0.23 & 2.67 & 2.44 & 0.16 & 1.87 & 0.19 & 0.15 \\
\hline BZT2c & 0.63 & 0.48 & 0.30 & 2.69 & 2.57 & 0.16 & 1.78 & 0.21 & 0.14 \\
\hline BZT2d & 0.47 & 0.38 & 0.37 & 1.51 & 2.58 & 0.55 & 47.4 & $\approx 0$ & 0.49 \\
\hline
\end{tabular}

In all cases, marked Stokes Shifts are observed (see $\Delta_{\text {SS }}$ in Table 1 and Figure 1), which are particularly large with compounds $\mathrm{T} 1$ and $\mathrm{T} 2$. This is due to the presence of donor and acceptor groups at the two terminal positions, which induce a charge-transfer character in the emissive species. It is wellknown that the dimethylamino group acts as a donor in aromatic compounds, while the strongly electron-accepting character of the nitro group is equally well-established.

Photophysical Parameters: Fluorescence Quantum Yields and Lifetimes.

It is interesting to compare T1 (synthetic precursor of BZT1b) and $\mathrm{T} 2$ which is also a precursor in terms of basic chromophoric unit compared to the other bithienyl derivatives. When T1 is compared with BZT1b, that is, the $\mathrm{CHO}$ group is replaced by the benzothiazole heterocycle, there are no significant differences between the spectral properties of the two compounds, suggesting very similar electron-accepting properties of these two groups in the ground state. However, this is not what happens with the photophysical parameters, where it can be seen (Table 2) that the $\phi_{\mathrm{F}}$ value increases by over an order of magnitude on going from T1 to BZT1b, that is, the benzothiazole unit enhances the radiative channel.

Fluorescence lifetimes were also obtained in solution and in the solid state (thin films) and the decays were, within our time resolution $(\sim 150 \mathrm{ps})$, seen to be single-exponential for all the compounds (see Table 2 and Figure 2). The values obtained were found to vary between $\approx 1$ ns and $\approx 4$ ns.

Inspection of Table 2 shows that the two precursors (T1 and T2), that is, the compounds without the benzothiazole unit, display the lowest fluorescence quantum yields of any of the compounds studied, although their fluorescence lifetimes are comparable to the other systems. Although triplet state formation can be seen to be an important pathway for excited-state deactivation of these two molecules $(\sim 10-15 \%$ of the overall quantum yields), it is clear that internal conversion is the dominant deactivation channel.

The arylthienyl-benzothiazole derivatives (BZT1a and BZT1b) were found to have the highest fluorescence yields of all the compounds studied in solution at $293 \mathrm{~K}$. Moreover, a direct comparison can be made between the $\alpha 1$-benzothiazole (BZT1a) and the $\alpha 2$-benzothiazole (BZT2b), showing that the replacement of a phenyl unit by a thienyl moiety leads to an increase of the fluorescence quantum yield. The bithienyl-benzothiazole compound BZT2a and its derivative BZT2d possessing the ethylenedioxy group show very similar spectral properties. However, as will be seen below, this is not true with the photophysical parameters.

The synthetic (T1) and the chromophoric (T2) precursors show the smallest separations between the HOMO and LUMO orbitals, which lead to red-shifts of the absorption and emission when compared with the thienyl-benzothiazole counterparts. This may be related to the greater electron-accepting ability of the end groups compared with the benzothiazole moiety. It is also interesting to note that the thienyl- and bithienyl-benzothiazoles studied and their precursors all present significantly higher $\phi_{\mathrm{F}}$ values than the corresponding oligothiophenes $\alpha 1$ (thiophene, effectively nonfluorescent) and $\alpha 2$ (bithiophene, $\phi_{\mathrm{F}}=0.026^{24}$ ).

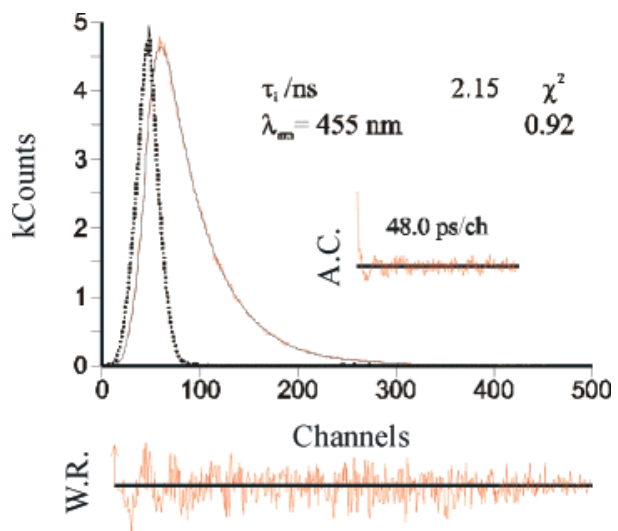

Figure 2. Fluorescence decay for BZT1a in ethanol at 293 K. The decay was fitted with a monoexponential law. For a better judgment of the quality of the fit, autocorrelation function (A.C.), weighted residuals (W.R.), and the chi-square value $\left(\chi^{2}\right)$ are also presented. 
Temperature Effect on the Spectra and Fluorescence Quantum Yields.

Decreasing the temperature leads to red-shifts in the absorption maxima of all compounds, with values of $\approx 30 \mathrm{~nm}$ for T1 and $\mathrm{T} 2$ and of $\approx 10 \mathrm{~nm}$ for the arylthienyl and bithienylbenzothiazoles, see Figure 1 and Table 1. The opposite behavior is found in fluorescence, where, in general, a blue-shift is observed upon cooling; this blue-shift in the emission spectra has been previously attributed to a difference in the most stable conformation within the potential energy curves for the $S_{1}$ and $\mathrm{S}_{0}$ states. $^{29}$

In contrast, for BZT2d, no marked spectral changes are observed on going from room temperature to low temperature and even to the solid state (see below). This suggests that, upon cooling, no significant structural changes are induced between the ground and excited states, possibly indicating a more rigid structure of this compound induced by the ethylenedioxy group. ${ }^{30}$

As can be seen from Table 2, with the exception of T1, T2, and BZT2a, the fluorescence quantum yields show a relatively modest decrease upon cooling from 293 to $77 \mathrm{~K}$. This decrease of the $\phi_{\mathrm{F}}$ values contrasts with the behavior of thiophene oligomers where an increase or constancy of this value is normally observed. ${ }^{31}$ This is in line with the behavior found for other oligomers such as the mixed penta-furan-thiophene ${ }^{32}$ and cruciform ${ }^{28}$ oligomers. The large increase in $\phi_{\mathrm{F}}$ on cooling observed with the oligothiophenes ${ }^{24}$ may be associated with their more flexible conformational structures, which favors nonradiative decay at room temperature, but which gets frozen out at lower temperatures. In contrast, the benzothiazole derivatives may possess a more rigid structure, making this pathway less important.

It is worth noting from the data in Table 2 that the increase of the $\phi_{\mathrm{F}}$ value by 1 order of magnitude in T1 and 2 orders of magnitude in T2 on going from RT to LT clearly seems to result from a reduction of the internal conversion channel due to the decreased coupling between the nonradiative modes of $S_{1}$ and $\mathrm{S}_{0}$. As discussed above, it seems reasonable that the behavior with these two compounds stems from torsional modes between the thiophene and $N, N$-dialkylaminophenyl in T1 or bithiophene and nitrophenyl groups in T2, coupling the nonradiative modes of the $S_{0}$ and $S_{1}$ states, which decrease at low temperatures as a consequence of decreased conformational mobility (or increased percentage of planar conformers).

\section{Solid-State Behavior.}

With thin films in a Zeonex matrix, from the absorption and emission spectra shown in Figures 1 and 2 and Table 1 it can be seen that there is little difference in the absorption maxima between the films and solutions at room temperature. However, this is not the case with fluorescence, where shifts are observed in spectra, together with changes in fluorescence quantum yields (Table 2), which, in general, are lower in the solid state relative to the $\phi_{\mathrm{F}}$ values at RT or in low-temperature glasses. Again, the exception involves the compounds $\mathrm{T} 1$ and $\mathrm{T} 2$, where an increase is found. This behavior is similar to that observed for $\pi$-conjugated oligomers and polymers and has been attributed to additional nonradiative decay channels due to intermolecular interactions in the solid state. ${ }^{33}$ The increase in $\phi_{\mathrm{F}}$ for $\mathrm{T} 1$ and $\mathrm{T} 2$ in the solid state probably has a similar explanation to the low-temperature values discussed above in terms of increased rigidity.

Transient Triplet-Triplet Spectra and Intersystem Crossing Quantum Yields.

The transient singlet-triplet difference absorption spectra of these compounds were studied by laser flash photolysis. The

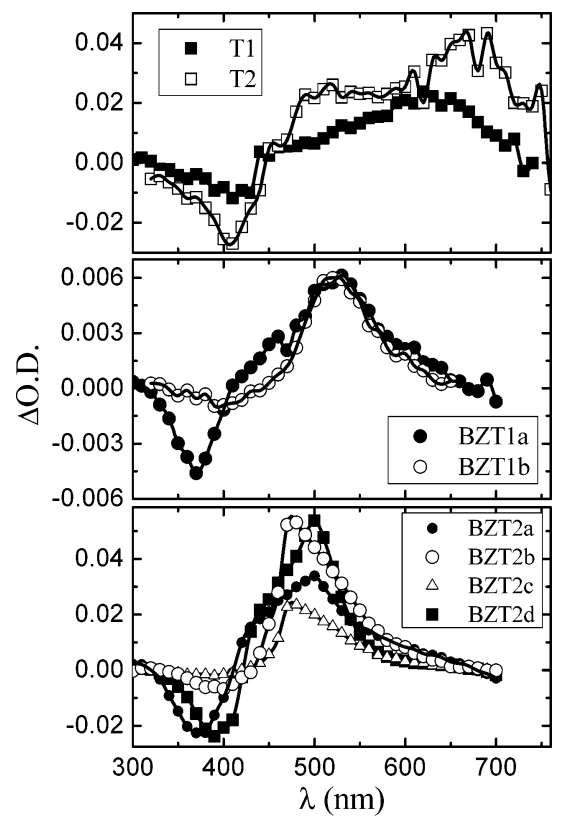

Figure 3. Transient singlet-triplet difference spectra for the arylthienyl- and bithienyl-benzothiazoles in ethanol at room temperature.

transient absorption spectra of these samples are broad (Figure 3 ), suggesting delocalization of the triplet excited state due to conjugation along the oligomer backbone. From Table 1 and Figure 3 it can be observed that compounds T1 and T2, which do not have the benzothiazole nucleus, have less localized triplet states. This can be attested to by the more than $200 \mathrm{~nm}$ difference between the $\mathrm{S}_{0} \rightarrow \mathrm{S}_{1}$ and $\mathrm{T}_{1} \rightarrow \mathrm{T}_{n}$ wavelength maxima in Table 1. It is relevant that we were unable to observe phosphorescence signals, which indicates a very efficient $\mathrm{T}_{1} \sim \sim \mathrm{S}_{0}$ radiationless process, as is also observed with the great majority of conjugated organic oligomers and polymers, including those with thiophene groups. ${ }^{24}$

\section{Yields of Singlet Oxygen Formation.}

Singlet oxygen formation quantum yields $\left(\phi_{\mathrm{D}}\right)$ were obtained following photolysis of aerated ethanol solutions of the compounds. Observation of the $\phi_{\Delta}$ values in Table 2 reveals that these are low with the exceptions of those for BZT2a and BZT2d. Although the yields of singlet oxygen formation are low with the other systems, in all cases the efficiency of ${ }^{1} \mathrm{O}_{2}{ }^{*}$ sensitization by the triplet state $\left(\mathrm{S}_{\Delta}\right)$ is always above $40 \%$, and in the great majority of the cases $\mathrm{S}_{\Delta} \approx 1$. These results clearly indicate energy transfer is efficient from the arylthienyl- or bithienyl-benzothiazoles triplet states to ground-state oxygen.

\section{Rate Constants.}

From the overall photophysical parameters (quantum yields and lifetimes), the rate constants for all decay processes can be obtained. These are shown in Table 3. From Table 3 it can be seen that the radiative rate constant ranges, for the compounds, lie between 0.234 and $0.362 \mathrm{~ns}^{-1}$, with the exception of the compounds $\mathrm{T} 1$ and $\mathrm{T} 2$ whose rate constants are 1-2 orders of magnitude lower.

For derivatives $\mathrm{T} 1$ and $\mathrm{T} 2$ the radiationless decay processes $\left(\phi_{\mathrm{IC}}\right.$ and $\left.\phi_{\mathrm{T}}\right)$ are the preferred pathways for excited-state deactivation, with internal conversion being the dominant decay process (see Table 2).

When a benzothiazole nucleus is introduced, and as a general trend for the arylthienyl and bithienyl-benzothiazole derivatives, the fluorescence deactivation channel tends to become dominant. However, competitive intersystem crossing is found for BZT2a 
TABLE 3: Rate Constants for the Arylthienyl- and Bithienyl-Benzothiazoles in Ethanol at Room Temperature $(293 \mathrm{~K})$ and in Films

\begin{tabular}{lcccccc}
\hline & $\begin{array}{c}k_{\mathrm{F}} \\
\left(\mathrm{ns}^{-1}\right)\end{array}$ & $\begin{array}{c}k_{\mathrm{NR}} \\
\left(\mathrm{ns}^{-1}\right)\end{array}$ & $\begin{array}{c}k_{\mathrm{IC}} \\
\left(\mathrm{ns}^{-1}\right)\end{array}$ & $\begin{array}{c}k_{\mathrm{ISC}} \\
\left(\mathrm{ns}^{-1}\right)\end{array}$ & $\begin{array}{c}k_{\mathrm{F}} \\
\left(\mathrm{ns}^{-1}\right)\end{array}$ & $\begin{array}{c}k_{\mathrm{NR}} \\
\left(\mathrm{ns}^{-1}\right) \\
(\mathrm{film})\end{array}$ \\
\hline T1 & 0.068 & 0.903 & 0.757 & 0.146 & 0.029 & 0.237 \\
T2 & 0.002 & 0.488 & 0.440 & 0.054 & 0.089 & 0.242 \\
BZT1a & 0.362 & 0.102 & 0.018 & 0.084 & 0.125 & 0.320 \\
BZT1b & 0.289 & 0.055 & 0.023 & 0.032 & 0.049 & 0.360 \\
BZT2a & 0.260 & 0.580 & 0.142 & 0.437 & 0.087 & 0.307 \\
BZT2b & 0.243 & 0.131 & 0.071 & 0.060 & 0.094 & 0.316 \\
BZT2c & 0.234 & 0.137 & 0.078 & 0.059 & 0.117 & 0.272 \\
BZT2d & 0.311 & 0.351 & $\approx 0$ & 0.364 & 0.143 & 0.244
\end{tabular}

and BZT2d, see Table 2. Indeed for BZT2a there is a marked increase in the intersystem crossing quantum yield $\left(\phi_{\mathrm{T}}\right)$ value at the expense of the radiative channel $\left(\phi_{\mathrm{F}}\right)$. From Table 2, it can also be seen that for BZT2d the internal conversion decay channel is an inefficient process $\left(\phi_{\mathrm{IC}} \approx 0\right)$. Comparison of data for BZT2a and BZT2d, that is, substitution of the $2,2^{\prime}$ bithiophene system by the $3^{\prime}, 4^{\prime}$-(ethylenedioxy)-2,2'-bithiophene shows that although there is an increase in the fluorescence quantum yield value (Table 2), the ISC yield is approximately constant, which means that the decrease in the fluorescence is made at the expense of an increase in the IC for BZT2a, Table 2 , which has the important consequence that the addition of the ethylenedioxy group appears to decouple the nonradiative modes of $S_{1}$ and $S_{0}$. In general, the introduction of alkoxyl endterminal groups in the bithiophene $(\alpha 2)$ moiety of the bithienylbenzothiazole systems, BZT2a versus BZT2b to BZT2c, significantly increases the $\phi_{\mathrm{F}}$ value at the expense nonradiative pathways $\left(\phi_{\mathrm{T}}\right.$ and $\phi_{\mathrm{IC}}$ in the case of BZT2d).

\section{Conclusions}

A comprehensive spectroscopic and photophysical investigation of arylthienyl- and bithienyl-benzothiazole derivatives has been made in solution and in the solid state (thin films). It is shown that the benzothiazole heterocycle strongly enhances the fluorescence excited-state deactivation channel. Comparison of the spectroscopic and photophysical properties is made with the unsubstituted parent compounds. The formyl-arylthiophene precursor (T1) and the bithienyl derivative (T2) display significantly lower fluorescence quantum yields at RT.

The results obtained for the bithienyl-benzothiazoles derivatives strongly suggest that having benzothiazole coupled to bithienyl units may be a powerful strategy for the design of more efficient OLEDs since it minimizes internal conversion, and significantly reduces triplet state formation.

Acknowledgment. Thanks are due to the Foundation for Science and Technology for financial support through the Centro de Química (Universidade do Minho). Financial support from FEDER and FCT (through projects POCI/QUI/55672/2004 and POCI/QUI/58291/ 2004) are also acknowledged. J.P. acknowledges FCT for a Ph.D. grant (SFRH/ BD/18876/2004).

Supporting Information Available: Synthesis and characterization and spectral and photophysical determinations. This material is available free of charge via the Internet at http:// pubs.acs.org.

\section{References and Notes}

(1) Milmo, S. Chem. Ind. 2007, 21.
(2) Roncali, J.; Frere, P.; Blanchard, P.; de Bettignies, R.; Turbiez, M.; Roquet, S.; Leriche, P.; Nicolas, Y. Thin Solid Films 2006, 511, 567.

(3) Colladet, K.; Fourier, S.; Cleij, T. J.; Lutsen, L.; Gelan, J.; Vanderzande, D.; Nguyen, L. H.; Neugebauer, H.; Sariciftci, S.; Aguirre, A.; Janssen, G.; Goovaerts, E. Macromolecules 2007, 40, 65.

(4) Blanchard, P.; Verlhac, P.; Michaux, L.; Frere, P.; Roncali, J. Chem. Eur. J. 2006, 12, 1244. 2659.

(5) Vamvounis, G.; Yu, J. F.; Holdcroft, S. Eur. Polym. J. 2004, 40,

(6) Gierschner, J.; Cornil, J.; Egelhaaf, H. J. Adv. Mater. 2007, 19, 173.

(7) Kulkarni, A. P.; Kong, X. X.; Jenekhe, S. A. J. Phys. Chem. B 2004, 108, 8689 .

(8) Jacob, J.; Sax, S.; Piok, T.; List, E. J. W.; Grimsdale, A. C.; Mullen, K. J. Am. Chem. Soc. 2004, 126, 6987.

(9) Granstrom, M. Polym. Adv. Technol. 1997, 8, 424.

(10) Pei, J.; Yu, W. L.; Huang, W.; Heeger, A. J. Acta Polym. 1999, $50,327$.

(11) Scherf, U. J. Mater. Chem. 1999, 9, 1853.

(12) Wang, X. J.; Andersson, M. R.; Thompson, M. E.; Ingandas, O. Thin Solid Films 2004, 468, 226.

(13) Zhang, X. H.; Wong, O. Y.; Gao, Z. Q.; Lee, C. S.; Kwong, H. L.; Lee, S. T.; Wu, S. K. Mater. Sci. Eng., B-Solid State Mater. Adv. Technol. 2001, 85, 182.

(14) Xu, X. J.; Liao, Y.; Yu, G.; You, H.; Di, C. G.; Su, Z. M.; Ma, D. G.; Wang, Q.; Li, S. Y.; Wang, S. Q.; Ye, J. P.; Liu, Y. Q. Chem. Mater. 2007, 19, 1740.

(15) Raposo, M. M. M.; Fonseca, A. M. C.; Kirsch, G. Tetrahedron 2004, 60, 4071 .

(16) Batista, R. M. F.; Costa, S. P. G.; Raposo, M. M. M. Tetrahedron Lett. 2004, 45, 2825.

(17) Costa, S. P. G.; Batista, R. M. F.; Cardoso, P.; Belsley, M.; Raposo, M. M. M. Eur. J. Org. Chem. 2006, 3938.

(18) Costa, S. P. G.; Batista, R. M. F.; Sousa, A. M. R. C.; Raposo, M. M. M. New fluorescent heterocyclic materials: Synthesis, solvatochrornic and fluorescence properties. In Advanced Materials Forum III, Pts 1 and 2; 2006; Mater. Sci. Forum, Trans Tech Publications: Zuerich, Switzerland, Vol. 514-516, pp 147.

(19) Batista, R. M. F.; Costa, S. P. G.; Malheiro, E. L.; Belsley, M.; Raposo, M. M. M. Tetrahedron 2007, 63, 4258

(20) Prasad, P. N.; Williams, D. J. Introduction to nonlinear optical effects in molecules and polymers; Wiley: New York, 1990.

(21) Albota, M.; Beljonne, D.; Bredas, J. L.; Ehrlich, J. E.; Fu, J. Y.; Heikal, A. A.; Hess, S. E.; Kogej, T.; Levin, M. D.; Marder, S. R.; McCordMaughon, D.; Perry, J. W.; Rockel, H.; Rumi, M.; Subramaniam, C.; Webb, W. W.; Wu, X. L.; Xu, C. Science 1998, 281, 1653.

(22) Oliva, M. M.; Casado, J.; Raposo, M. M. M.; Fonseca, A. M. C.; Hartmann, H.; Hernandez, V.; Navarrete, J. T. L. J. Org. Chem. 2006, 71, 7509.

(23) Vaschetto, M. E.; Retamal, B. A. J. Phys. Chem. A 1997, 101 6945.

(24) Becker, R. S.; Seixas de Melo, J.; Maçanita, A. L.; Elisei, F. J. Phys. Chem. 1996, 100, 18683

(25) Murov, S.; Charmichael, I.; Hug, G. L. Handbook of Photochemistry; M. Dekker Inc.: New York, 1993.

(26) Pina, J.; Burrows, H. D.; Becker, R. S.; Dias, F. B.; Maçanita, A. L.; Seixas de Melo, J. J. Phys. Chem. B 2006, 110, 6499.

(27) Striker, G.; Subramaniam, V.; Seidel, C. A. M.; Volkmer, A. $J$ Phys. Chem. B 1999, 103, 8612

(28) Pina, J.; Seixas de Melo, J.; Burrows, H. D.; Bilge, A.; Farrell, T.; Forster, M.; Scherf, U. J. Phys. Chem. B 2006, 110, 15100.

(29) Seixas de Melo, J.; Pina, J.; Burrows, H. D.; Brocke, S.; Herzog, O.; Thorn-Csanyi, E. Chem. Phys. Lett. 2004, 388, 236.

(30) Gao, D. Q.; Kohler, B.; Scherf, U. J. Phys. Chem. B 2006, 110, 24346.

(31) Seixas de Melo, J.; Pina, J.; Burrows, H. D.; Di Paolo, R. E.; Maçanita, A. L. Chem. Phys. 2006, 330, 449.

(32) Seixas de Melo, J.; Elisei, F.; Becker, R. S. J. Chem. Phys. 2002, 117,4428

(33) Greenham, N. C.; Samuel, I. D. W.; Hayes, G. R.; Phillips, R. T.; Kessener, Y.; Moratti, S. C.; Holmes, A. B.; Friend, R. H. Chem. Phys. Lett. 1995, 241, 89 . 